\title{
Path integral formulation of fractional Brownian motion for general Hurst exponent
}

\author{
I Calvo ${ }^{1}$ and R Sánchez ${ }^{2}$ \\ ${ }^{1}$ Laboratorio Nacional de Fusión, Asociación EURATOM-CIEMAT, 28040 Madrid, \\ Spain \\ ${ }^{2}$ Fusion Energy Division, Oak Ridge National Laboratory, Oak Ridge, TN 37831, \\ U.S.A. \\ E-mail: ivan.calvo@ciemat.es, sanchezferlr@ornl.gov
}

\begin{abstract}
In J. Phys. A: Math. Gen. 28, 4305 (1995), K. L. Sebastian gave a pathintegral computation of the propagator of subdiffusive fractional Brownian motion (fBm), i.e. fBm with a Hurst or self-similarity exponent $H \in(0,1 / 2)$. The extension of Sebastian's calculation to superdiffusion, $H \in(1 / 2,1]$, becomes however quite involved due to the appearance of additional boundary conditions on fractional derivatives of the path. In this paper, we address the construction of the path integral representation in a different fashion, which allows to treat both subdiffusion and superdiffusion on an equal footing. The derivation of the propagator of $\mathrm{fBm}$ for general Hurst exponent is then performed in a neat and unified way.

PACS numbers: 05.40.-a, 02.50.Ey, 05.10.Gg
\end{abstract}

\section{Introduction}

The Langevin representation for Brownian motion has been known for a long time [1, 2]. In one dimension, it can be formally written as

$$
x(t)=x_{0}+\int_{0}^{t} \xi\left(t^{\prime}\right) \mathrm{d} t^{\prime},
$$

where $\xi(t)$ is a Gaussian, uncorrelated noise function:

$$
\left\langle\xi(t) \xi\left(t^{\prime}\right)\right\rangle=\delta\left(t-t^{\prime}\right)
$$

The propagator of Brownian motion, which gives the probability of finding the Brownian walker at some location $x$ at time $t>0$ after having started at $x_{0}$ at time 0 , is a Gaussian whose width grows as $t^{1 / 2}$ :

$$
G\left(x, t \mid x_{0}, 0\right)=\frac{1}{\sqrt{2 \pi t}} \exp \left(-\frac{\left(x-x_{0}\right)^{2}}{2 t}\right) .
$$

However, many interesting processes (see [3] for a review) ranging from hydrology [4] to finance [5] or tracer transport by turbulent flows [6] exhibit anomalous diffusion, $\left\langle\left(x-x_{0}\right)^{2}\right\rangle \propto t^{2 H}$, with $H \neq 1 / 2$. In many situations the anomalous behaviour is 
due to spatio-temporal correlations, which translate into long-term time correlations in Lagrangian quantities. A generalization of Brownian motion which was proposed to include such correlations is known as fractional Brownian motion (fBm) [7]. Although slightly different representations can be found in the literature, the one more convenient for our purposes is

$$
x(t)=x_{0}+\frac{1}{\Gamma\left(H+\frac{1}{2}\right)} \int_{0}^{t}\left(t-t^{\prime}\right)^{H-\frac{1}{2}} \xi\left(t^{\prime}\right) \mathrm{d} t^{\prime},
$$

where $\Gamma(\cdot)$ is the Gamma function and $\xi(t)$ is still Gaussian and uncorrelated $\dagger$. The exponent $H$ corresponds to both the self-similarity exponent of the process [7] and the Hurst exponent [10] of the incremental process $\mathrm{d} x(t):=x(t+\mathrm{d} t)-x(t)$. On the one hand, the average motion is invariant under the transformation $(x, t) \rightarrow\left(\mu^{H} x, \mu t\right)$. On the other hand, the increments $\mathrm{d} x(t)$ are uncorrelated for $H=1 / 2$ and anticorrelated (correlated) for $H \in(0,1 / 2)(H \in(1 / 2,1])$. Consequently, (4) can be used to model diffusive $(H=1 / 2)$, subdiffusive $(H \in(0,1 / 2))$, and superdiffusive $(H \in(1 / 2,1])$ transport processes.

In a beautiful paper [11], K. L. Sebastian showed that it is very advantageous to rewrite $\mathrm{fBm}$ in terms of fractional differential operators,

$$
x(t)=x_{0}+{ }_{0} D_{t}^{-(H+1 / 2)} \xi,
$$

where ${ }_{0} D_{t}^{-\alpha}$ is the Riemann-Liouville fractional integral of order $\alpha$ [12, 13]. Indeed, by combining path integral methods [14] with fractional calculus, he computed the exact propagator of (41) for $H \leq 1 / 2$. However, the case $H>1 / 2$ is not addressed in [11] because the problem becomes quite more complicated from the technical point of view. The difficulties may be explained as follows. Sebastian constructs the path integral by inverting (5) in terms of Riemann-Liouville fractional differential operators. This is easy for $H \leq 1 / 2$ because such inversion is well-defined simply imposing the boundary conditions $x(0)=x_{0}$ and $x(T)=x_{T}$. However, if $H>1 / 2$ additional boundary conditions involving fractional derivatives at $t=0$ are required. Not only is the physical meaning of these 'fractional initial conditions' unclear, but also the computation along the lines of [11] results too cumbersome.

In this paper, we formulate the path integral for fBm using instead $\xi(t)$ as the integration variable. This choice avoids the need to invert (5), and thus the need for the additional fractional initial conditions. In our approach the difficulty resides in how to manage the boundary conditions of $x(t)$, which translate into a non-local constraint on the admissible realizations of the noise. We will see below that the usage of Lagrange multipliers allows to do this in an easy and transparent way, thus providing a general path-integral derivation of the well-known propagator of fBm [15].

$\ddagger$ In [7, 8] fractional Brownian motion is defined by (4) except that the lower limit of the integral is taken at $-\infty$ and the issue of boundary conditions is not present. Our definition is better suited for the class of applications we are interested in (e.g. analysis of series of Lagrangian velocities in turbulent plasmas). For such physical problems, the stochastic differential equation is naturally formulated in a domain $t \in\left(t_{0}, \infty\right)$, with finite $t_{0}$. An equation analogous to (4) was used in [9], where $\mathrm{fBm}$ and fractional time processes are compared. 


\section{Path integral calculation of the propagator}

Given a Langevin equation describing the motion of a particle, the propagator $G\left(x_{T}, T \mid x_{0}, 0\right)$ is defined as the probability to find the particle at $x=x_{T}$ at time $t=T$ if initially, $t=0$, it was located at $x=x_{0}$. Hence, the propagator can be viewed as the motion of the particle averaged over all realizations of the noise compatible with the boundary conditions $x(0)=x_{0}, x(T)=x_{T}$. In the path integral formalism this is formulated as follows. The essential object is the probability measure $\mathcal{P}(\xi(t)) \mathcal{D} \xi(t)$ on the space of maps $\xi(t):[0, T] \rightarrow \mathbb{R}$. Since $\xi(t)$ is uncorrelated in time and distributed as a Gaussian for each $t$, this is naturally defined as

$$
\mathcal{P}(\xi(t)) \mathcal{D} \xi(t)=\exp \left(-\frac{1}{2} \int_{0}^{T} \xi(t)^{2} \mathrm{~d} t\right) \mathcal{D} \xi(t)
$$

We want to compute the propagator of (5). Firstly, note that the boundary conditions $x(0)=x_{0}, x(T)=x_{T}$ translate into the following constraint on $\xi(t)$ :

$$
{ }_{0} D_{T}^{-(H+1 / 2)} \xi=x_{T}-x_{0} .
$$

Therefore, the propagator can be written as the expectation value:

$G\left(x_{T}, T \mid x_{0}, 0\right)=\int \delta\left({ }_{0} D_{T}^{-(H+1 / 2)} \xi-\left(x_{T}-x_{0}\right)\right) \exp \left(-\frac{1}{2} \int_{0}^{T} \xi(t)^{2} \mathrm{~d} t\right) \mathcal{D} \xi(t)$,

where the Dirac delta function ensures that we only integrate over maps $\xi(t)$ satisfying (7).

We proceed to compute the path integral on the right-hand side of (8). Define the action

$$
S[\xi(t)]=\frac{1}{2} \int_{0}^{T} \xi(t)^{2} \mathrm{~d} t,
$$

and consider infinitesimal variations on the space of maps $\xi(t)$ satisfying (77). We denote by $\bar{\xi}(t)$ the map which makes the action stationary under such variations. Observe that, in particular, $\bar{\xi}(t)$ verifies (7). Now, we perform the following change of variables in (8):

$$
\xi(t)=\bar{\xi}(t)+\eta(t) .
$$

The constraint (7) implies that

$$
{ }_{0} D_{T}^{-(H+1 / 2)} \eta=0 .
$$

Using that the action is quadratic in $\xi(t)$ and that $\bar{\xi}(t)$ makes it stationary,

$$
G\left(x_{T}, T \mid x_{0}, 0\right)=e^{-S[\bar{\xi}(t)]} \int \delta\left({ }_{0} D_{T}^{-(H+1 / 2)} \eta\right) e^{-S[\eta(t)]} \mathcal{D} \eta(t),
$$

whence we deduce that the propagator is of the form

$$
G\left(x_{T}, T \mid x_{0}, 0\right)=f(T) e^{-S[\bar{\xi}(t)]} .
$$

The function $f(T)$ will be determined at the end of the calculation by imposing the normalization condition

$$
\int_{-\infty}^{\infty} G\left(x_{T}, T \mid x_{0}, 0\right) \mathrm{d} x_{T}=1, \forall T .
$$


It only remains to compute the stationary points of $S[\xi(t)]$ subject to the constraint (7). It is difficult to do it directly, for the condition (7) is hard to implement. The technique of Lagrange multipliers saves the day, though. Our problem is equivalent to finding the stationary points of

$$
\tilde{S}[\xi(t), \lambda]=\frac{1}{2} \int_{0}^{T} \xi(t)^{2} \mathrm{~d} t+\lambda\left({ }_{0} D_{T}^{-(H+1 / 2)} \xi-\left(x_{T}-x_{0}\right)\right),
$$

under arbitrary infinitesimal variations of $\xi(t)$ and the Lagrange multiplier $\lambda \in \mathbb{R}$.

From variations with respect to $\lambda$,

$$
\delta_{\lambda} \tilde{S}[\xi(t), \lambda]=\delta \lambda\left({ }_{0} D_{T}^{-(H+1 / 2)} \xi-\left(x_{T}-x_{0}\right)\right),
$$

we recover, of course, the constraint (7):

$$
{ }_{0} D_{T}^{-(H+1 / 2)} \bar{\xi}=x_{T}-x_{0} .
$$

Let us perform now variations in $\xi(t)$ :

$$
\delta_{\xi} \tilde{S}[\xi(t), \lambda]=\int_{0}^{T} \xi(t) \delta \xi(t) \mathrm{d} t+\lambda_{0} D_{T}^{-(H+1 / 2)} \delta \xi .
$$

Writing explicitly the fractional integral:

$$
\begin{aligned}
\delta_{\xi} \tilde{S}[\xi(t), \lambda] & =\int_{0}^{T} \xi(t) \delta \xi(t) \mathrm{d} t+\frac{\lambda}{\Gamma(H+1 / 2)} \int_{0}^{T}(T-t)^{H-1 / 2} \delta \xi(t) \mathrm{d} t \\
& =\int_{0}^{T}\left(\xi(t)+\frac{\lambda}{\Gamma(H+1 / 2)}(T-t)^{H-1 / 2}\right) \delta \xi(t) \mathrm{d} t .
\end{aligned}
$$

Since $\delta_{\xi} \tilde{S}[\bar{\xi}(t), \lambda]$ must vanish for arbitrary $\delta \xi(t)$, we deduce

$$
\bar{\xi}(t)+\frac{\lambda}{\Gamma(H+1 / 2)}(T-t)^{H-1 / 2}=0
$$

The Lagrange multiplier is determined by applying ${ }_{0} D_{T}^{-(H+1 / 2)}$ to Eq. (21) and using (17):

$$
x_{T}-x_{0}+\frac{\lambda}{\Gamma(H+1 / 2)^{2}} \int_{0}^{T}(T-t)^{2 H-1} \mathrm{~d} t=0 .
$$

Immediately, we find

$$
x_{T}-x_{0}+\frac{\lambda}{\Gamma^{2}(H+1 / 2)} \frac{T^{2 H}}{2 H}=0,
$$

and

$$
\lambda=-2 H \Gamma^{2}(H+1 / 2) \frac{x_{T}-x_{0}}{T^{2 H}} .
$$

Inserting this in (21):

$$
\bar{\xi}(t)=2 H \Gamma(H+1 / 2) \frac{x_{T}-x_{0}}{T^{2 H}}(T-t)^{H-1 / 2} .
$$

Going back to (13) we straightforwardly obtain

$G\left(x_{T}, T \mid x_{0}, 0\right)=f(T) \exp \left(-H \Gamma^{2}(H+1 / 2) \frac{\left(x_{T}-x_{0}\right)^{2}}{T^{2 H}}\right)$. 
And $f(T)$ is obtained from the normalization property, (14):

$$
f(T)=\sqrt{\frac{H}{\pi}} \frac{\Gamma(H+1 / 2)}{T^{H}} .
$$

Hence, finally,

$$
G\left(x_{T}, T \mid x_{0}, 0\right)=\sqrt{\frac{H}{\pi}} \frac{\Gamma(H+1 / 2)}{T^{H}} \exp \left(-H \Gamma^{2}(H+1 / 2) \frac{\left(x_{T}-x_{0}\right)^{2}}{T^{2 H}}\right) .
$$

\subsection{The kinetic equation}

Once the propagator is known, we can easily derive the kinetic equation associated to $\mathrm{fBm}$. The Fourier transform of $G\left(x, t \mid x_{0}, 0\right)$ with respect to $x$ is

$$
\hat{G}(k, t)=\exp \left(-\frac{t^{2 H}}{4 H \Gamma^{2}(H+1 / 2)} k^{2}\right) .
$$

Differentiating with respect to $t$ :

$$
\partial_{t} \hat{G}(k, t)=-\frac{t^{2 H-1}}{2 \Gamma^{2}(H+1 / 2)} k^{2} \hat{G}(k, t) .
$$

Finally, we Fourier invert this expression to get the kinetic equation:

$$
\partial_{t} G(x, t)=\frac{t^{2 H-1}}{2 \Gamma^{2}(H+1 / 2)} \partial_{x}^{2} G(x, t),
$$

which is a diffusion equation with time-dependent diffusivity, as originally deduced in [15] from arguments based on the fluctuation-dissipation theorem. The invariance of (31) under the transformation $(x, t) \rightarrow\left(\mu^{H} x, \mu t\right)$ is manifest. Obviously, when $H=1 / 2$ we retrieve the standard diffusion equation associated to Brownian motion:

$$
\partial_{t} G(x, t)=\frac{1}{2} \partial_{x}^{2} G(x, t)
$$

\section{Conclusions}

In this paper we have computed by path integral methods the well-known propagator of fBm for general Hurst exponent, extending the construction of [11], which was restricted to the subdiffusive case. The key technical point in our approach is to use the noise as path integral variable instead of the coordinate of the particle trajectory.

Acknowledgements: I. C. acknowledges the hospitality of Oak Ridge National Laboratory, were this work was carried out. Part of this research was sponsored by the Laboratory Research and Development Program of Oak Ridge National Laboratory, managed by UT-Battelle, LLC, for the US Department of Energy under contract number DE-AC05-00OR22725. 


\section{References}

[1] P. Langevin. C. R. Acad. Sci. Paris, 146:530-533, 1908.

[2] G. E. Uhlenbeck and L. S. Ornstein. Phys. Rev., 36:823-841, 1930.

[3] R. Metzler and J. Klafter. J. Phys. A: Math. Gen., 37:R161-R208, 2004.

[4] F. J. Molz, H. H. Liu, and J. Szulga. Water Resour. Res., 33:2273, 1997.

[5] R. J. Elliott and J. van der Hoek. Fractional Brownian motion and financial modeling. In Trends in Mathematics, Birkhäuser Verlag, Basel, 2001.

[6] R. Sánchez, B. A. Carreras, D. E. Newman, V. E. Lynch, and B. Ph. van Milligen. Phys. Rev. E, 74:016305, 2006.

[7] B. B. Mandelbrot and J. W. van Ness. SIAM Rev., 10:422, 1968.

[8] G. Samorodnitsky and M. S. Taqqu. Stable non-Gaussian processes. Chapman \& Hall, New York, 1994.

[9] E. Lutz. Phys. Rev. E, 64:051106, 2001.

[10] H. E. Hurst. Trans. Am. Soc. Civ. Eng., 116:770, 1951.

[11] K. L. Sebastian. J. Phys. A: Math. Gen., 28:4305, 1995.

[12] K. Oldham and J. Spanier. The Fractional Calculus. Academic Press, New York, 1974.

[13] I. Podlubny. Fractional differential equations. Academic Press, New York, 1998.

[14] R. P. Feynman and A. R. Hibbs. Quantum Mechanics and Path Integrals. McGraw-Hill, New York, 1965.

[15] K. G. Wang and C. W. Lung. Phys. Lett. A, 151:119, 1990. 